\title{
Continuing metamorphosis of evidence-based medicine; the past, present and the future
}

\author{
Asoka Gunaratne ${ }^{1}$, Umesh Jayarajah ${ }^{2}$, Anuruddha M. Abeygunasekera ${ }^{2}$ \\ ${ }^{I}$ Department of Anaesthesiology, ${ }^{2}$ Department of Urology, Colombo South Teaching Hospital, Kalubowila, Sri Lanka.
}

\author{
Correspondence: Dr. Asoka Gunaratne \\ e-mail: asoka.gunaratne@yahoo.com \\ https://orcid.org/0000-0003-2628-8877 \\ Submitted on 01.11.2020 and accepted for publication on 15.11.2020
}

Evidence-based medicine (EBM) is a relatively new concept coined in the 1990s (1). It is considered a systemic approach to assess the best available published research as the foundation of clinical decision making. At first, EBM was defined as a substitute for clinical experience, however, later definitions realised its complementary nature to enhance and assist clinical experience (2). In 1996, Sacket et al., formerly defined EBM as "the conscientious and judicious use of current best evidence from clinical care research in the management of individual patients" (1).

There are some accounts to suggest that there were clinical practices based on evidence during the ancient period (1). During the renaissance era of $\mathrm{EBM}$, i.e. in the $17^{\text {th }}$ century, personal journals and textbooks were given more emphasis (1). The period of $1900-1970$ is known as the transitional era of EBM, during which well formulated textbooks and peer-reviewed journals started to flourish and led to the modern era of EBM after 1970s (1). Technology, the internet, computers and databases enabled access to large amount of information and fast-forwarded the development and progression of EBM in the modern era (1).

Medicine is and has always been associated with lack of precision and uncertainty. This is seen at a greater scale that the infamous conflict in Vietnam which is used as an example for this phenomenon. Doctors dealing complex clinical issues based on EBM or assessing a trainee, based on portfolio or even assessing the candidate by means of examinations may all be examples of the "McNamara fallacy"(3).
Robert McNamara was an economist who served the US Army's Department of Statistical Control during the Second World War. He applied rigorous statistical methodology to the planning of aerial bombing, achieving a dramatic improvement in efficiency. In the administration of President John F. Kennedy, McNamara was appointed the Secretary of Defence. During the conflict in Vietnam, McNamara once again applied his statistical modeling. He believed that as long as his statistical model which was predominantly based on quantitative measurements was favourable, the war would be eventually won. McNamara's "quantitative" style relied heavily on the numbers calculated by computers and ignored other human dimensions such as popular views and attitudes in South Vietnam. Although some were against his views, he disregarded them and believed that his numbers were infallible and his judgment based on the numbers was always correct (3).

The South Vietnamese Army reported what they thought the US wanted to hear. Americans never questioned the numbers. However, they failed to realise the limitations of high-tech military equipment, numerous forces and traditional military doctrines when fighting an unconventional war without battle lines. The calculated figures were wrong and all projections made on that were equally wrong. US forces had to face a never-ending war with heavy casualties. Finally, they had to withdraw from the war and McNamara resigned from his post. This phenomenon of danger in over-reliance on statistics was used to coin the phrase "McNamara fallacy" by Danial Yankelowich in 1972 (3). 
The McNamara fallacy in medicine is characterised by the over reliance on crude metrics such as mortality rates, the delusion that all complexities can yield numerical analysis, setting arbitrary targets which do not necessarily improve patient care and the neglect of unquantifiable attributes such as communication and competence. In the research setting, it is acceptable to measure what can be measured. However, disregarding what is not easily measured as unimportant is both artificial and misleading. Assuming what cannot be measured as non-existing is suicidal!

Tonelli stated that, EBM has changed the perception of clinical judgment in medicine (4). Deviations form EBM guidelines are given an immediate disapproval by the peers until proper justification is given (4). This has led to a practice known as defensive medicine, as the clinicians often act to avoid liability (4). In a court of law, arguing and citing their personal experience with similar patients will not be as good as citing a study from a prestigious journal, whether the information is directly applicable for the concerned patient or not (4). Tonelli further warned that, although EBM may improve positive outcomes in a large population, it never promises "the best decision in a particular situation"(4).

Statistical significance does not always denote clinical significance. In 2015, the editors of Basic and Applied Social Psychology banned the " $p$ " value concept in their journal (5). It is imperative to understand that $p$-value is a crude measure of probability or certainty and not the ultimate truth. Importantly, the cut off of 0.05 is arbitrary and has minimal scientific basis (6). In 2016, the American Statistical Association (ASA) declared "scientific conclusions and business or policy decisions should not be based on the $p$-value" (7). It would be foolish to argue that metrics have no place in medicine. However, overemphasis on such metrics has distracted contemporary medicine from its core mission. The obsession with metrics is partly due to the managerialism and one should not be in a delusion that generic business methods can be easily applied to the complexities of healthcare. This has led to a novel shift from evidence-based medicine (EBM) to more individual patient-focused precision medicine.
A critical analysis of evidence should be performed before considering suitable for EBM. These may include factors such as the characteristics of the study population in comparison to the local context, inclusion and exclusion criteria, adherence to protocol, independent assessments and bias, statistics: appropriateness and clinical significance, conflict of interests of authors and the pragmatic nature of the study. However, as clinicians and scientists, we should be aware that there are numerous known immeasurable factors and a cosmos of unknown factors which we cannot comprehend that may determine the outcome. Most of these variables that matter cannot be measured. Therefore, management protocol of one patient cannot be the same for the next patient with the same label of a clinical condition. Unfortunately, guidelines and protocols which forms the bedrock of EBM are based mainly on statistics and do not allow these variables to be considered. Some of these include socioeconomic, cultural, spiritual factors, the patients' beliefs and goals, cost and resource limitations.

At examinations and during clinical practice doctors are taught to adhere to these guidelines. Examiners study guidelines before the examination and question the candidates the next day! Final products of such training provide less optimal patient care and decision making in real time clinical practice. Guidelines are only to guide medical personnel. Unfortunately, it has become the holy grail of medical practice. Therefore, during training, this has to be emphasised. At the end of each guideline, it is important to stress the fact that although it should form the basis of patient care, there are many variables that could matter in the final decision making related to an individual patient and such factors should be considered wisely before the final care pathway is formulated. For this, the clinician should have mindfulness, experience and wisdom.

EBM is solely based on available best research findings. The hierarchy of evidence is determined mainly by statistics. However, statistics can be made complex and manoeuvered the way the experts want (8). The fundamental concept in statistics is to lump data. In medicine this data has to be as homogenous as possible. However much we try to cluster a cohort with similar clinical parameters, there is enough heterogeneity within 
these said to be homogenous group which are inseparable with number. We should get rid of the delusion that all complexities can yield numerical analysis. Clinical competence is one such example. Neglect of unquantifiable attributes and the use of wrong end points that do not necessarily have a clinical benefits are all examples of McNamara fallacy in medicine. Most clinicians who gather evidence based on published research do not have a masterly knowledge on statistics to identify its pitfalls. Therefore, it is important to accept uncertainty as a possibility in research. Do not rely always on numbers. Some who are not good and familiar in statistics, should be careful during interpretation of study results and others' comments based on statistics. We must learn to look at the bigger picture. We need to train our undergraduates and postgraduates to look at the bigger picture while not forgetting the microcosm. Guidelines ignore the bigger picture most of the time. Always tailor your management plan to the individual in front of you in this place, rather than to all men and women in the world with the same illness.

EBM was conceptualised to improve clinical decision making. It was hoped that improved clinical decision making would lead to improved health outcomes. However, improved outcome is not merely improved mortality, morbidity and quality of life. It encompasses increased efficiency of clinical care at individual, institutional and health care system levels (9). To achieve these goals, we need to go beyond EBM (10). EBM is an excellent concept when specific diseases are discussed in lecture halls, books and journals. It certainly helps an inexperienced junior doctors to practice some kind of standardised medicine with uniformity at a basic level. However, when it comes to an individual patient, the attending clinician needs to take a step ahead and tailor the most appropriate treatment plan combining his/ her experience, many other identifiable factors that affect management of this particular patient at this moment in this place. Those who provide the same treatment based on EBM in its entirety for every patient with the same label of a given disease will provide suboptimal care to his patient because it will not lead to increased efficiency of clinical care at individual, institutional and healthcare system levels. This has to be conveyed to all undergraduates and postgraduates who consider guidelines found in their smart phones as the gospel truth. Thought practice of medicine in a personalised way specific to a given patient requires substantial clinical experience and wisdom, junior doctors should be made aware of this alternative to standard EBM.

The future physicians will act as knowledge administrators, not knowledge accumulators. They should perform better patient interaction, be compassionate, consult the patient regarding his/ her problem and provide assistance in the decisionmaking process (11). They should be mindful of the applicability of EBM to the individual patients and should be aware of the unknown and immeasurable factors that may alter outcomes with insight on the pitfalls of EBM.

Conflicts of interest: Authors have no conflicts of interest.

Funding: None

\section{References}

1. Claridge JA, Fabian TC. History and development of evidence-based medicine. World Journal of Surgery. 2005; 29(5): 547-53.

2. Siwek J. Evidence-Based Medicine: Common Misconceptions, Barriers, and Practical Solutions. American Family Physician. 2018; 98(6): 343-4.

3. O'Mahony S. Medicine and the McNamara fallacy. The Journal of the Royal College of Physicians of Edinburgh. 2017; 47(3): 281-7.

d. Goldman JJ, Shih TL. The limitations of evidence-based medicine: applying population-based recommendations to individual patients. AMA Journal of Ethics. 2011; 13(1): 26-30.

5. Trafimow D, Marks M. Editorial. Basic and Applied Social Psychology. 2015;37(1): 1-2.

6. Andrade C. The P Value and Statistical Significance: Misunderstandings, Explanations, Challenges, and Alternatives. Indian Journal of Psychological Medicine. 2019; 41(3): 210-5. 
7. Wasserstein RL, Lazar NA. The ASA statement on $p$-values: context, process, and purpose. Taylor \& Francis. 2016.

8. Bhatt S. Time to reconsider our obsession with statistical significance. Education for Health. 2019; 32(3): 135-136.

9. Christmas C, Kravet SJ, Durso SC, Wright SM, editors. Clinical excellence in academia: perspectives from masterful academic clinicians. Mayo Clinic Proceedings. 2008; 83(9): 989-994.
10. Abeygunasekera AM. Beyond evidence-based medicine: Need for a new paradigm in patient care delivery. Education for Health. 2014; 27(3): 302-302.

11. You S. Perspective and future of evidence-based medicine. Stroke and Vascular Neurology. 2016; 1(4): 161-164. 